Check for updates

Cite this: Chem. Commun., 2017, 53, 10946

Received 28th July 2017,

Accepted 7th August 2017

DOI: 10.1039/c7cc05906j

rsc.li/chemcomm

\section{Decoration of exfoliated black phosphorus with nickel nanoparticles and its application in catalysis $\uparrow$}

\author{
Maria Caporali, (D) *a Manuel Serrano-Ruiz, (D) ${ }^{a}$ Francesca Telesio, (D) b \\ Stefan Heun, (D) ${ }^{b}$ Giuseppe Nicotra, (D) ${ }^{c}$ Corrado Spinella (D) $^{c}$ and \\ Maurizio Peruzzini (D) *a
}

\begin{abstract}
Nickel nanoparticles were dispersed on the surface of exfoliated black phosphorus and the resulting nanohybrid Ni/2DBP showed an improved stability with respect to pristine 2D BP when kept under ambient conditions in the dark. Ni/2DBP was applied as a catalyst in the semihydrogenation of phenylacetylene and exhibited high conversion and selectivity towards styrene. These features were preserved after recycling tests revealing the high stability of the nanohybrid.
\end{abstract}

Black phosphorus (BP) has a layered structure analogue to graphite, and lately it has been shown that, following a similar procedure to that of bulk graphite, it can be exfoliated down to the monolayer. In this way, a new member of the growing family of 2D materials, named phosphorene, ${ }^{1,2}$ the all P-counterpart of graphene, was isolated. Single- and few-layer BP (2D BP) have been obtained by either micromechanical cleavage (Scotch tape method) $)^{3}$ or liquid exfoliation. ${ }^{4}$ In our labs, good quality phosphorene flakes were prepared by sonicating BP microcrystals in dimethylsulfoxide (DMSO) under an inert atmosphere in the dark; ${ }^{5}$ see Fig. S1-S4 (ESI $\dagger$ ) for the STEM and EELS characterization.

Since its discovery, the new P-based 2D material has attracted enormous interest, as it is endowed with a direct and tunable band gap ranging from $0.3 \mathrm{eV}$ (bulk) to $2.0 \mathrm{eV}$ (monolayer) and a high carrier mobility (up to $6000 \mathrm{~cm}^{2} \mathrm{~V}^{-1} \mathrm{~s}^{-1}$ ), which prompted its application towards the production of nano-electronic devices, such as field effect transistors (FETs). ${ }^{7}$ In spite of this huge interest, only an handful of reports have dealt with the surface functionalization of 2D BP and its application in chemical processes so far. ${ }^{8}$ Based on first-principles calculations, a systematic study on the binding energy, geometry, magnetic moment, and electronic structure of several metal ad-atoms adsorbed on phosphorene has been carried out, ${ }^{9}$ predicting that the immobilization of transition

\footnotetext{
${ }^{a}$ CNR-ICCOM, Via Madonna del Piano 10, 50019 Sesto Fiorentino, Italy.

E-mail: maria.caporali@iccom.cnr.it, maurizio.peruzzini@iccom.cnr.it

${ }^{b}$ NEST, Istituto Nanoscienze-CNR and Scuola Normale Superiore,

Piazza S. Silvestro 12, 56127 Pisa, Italy

${ }^{c}$ CNR-IMM, Istituto per la Microelettronica e Microsistemi, Strada VIII, 5, 95121 Catania, Italy

† Electronic supplementary information (ESI) available. See DOI: 10.1039/c7cc05906j
}

metals on the surface of phosphorene is feasible, while preserving its structural integrity. On this basis, we added dropwise a colloidal solution of nickel nanoparticles ${ }^{10}$ to a suspension of $2 \mathrm{D}$ BP under vigorous stirring. The reaction was monitored by UV-Vis spectrophotometry. Spectra were acquired first on colloidal Ni NPs and on a suspension of 2D BP, giving two distinct behaviours; see Fig. 1. Running UV-Vis right after mixing the two suspensions, the dispersion curve characteristic of colloidal Ni NPs disappeared, suggesting that Ni NPs were immobilized on the surface of BP.

TEM analysis showed well-dispersed nickel nanoparticles on the surface of black phosphorus nanosheets; see Fig. 2 and Fig. S8 (ESI $\dagger$ ). After immobilization, Ni nanoparticles preserved their size, average diameter of $11.9 \pm 0.8 \mathrm{~nm}$, and no aggregation took place, see for comparison Fig. S5 (ESI $\dagger$ ) for TEM image and size distribution of bare colloidal Ni NPs.

EDX performed on different points of the same sample confirmed the chemical identity of the 2D material and corroborated the presence of $\mathrm{Ni}$ and elemental $\mathrm{P}$ without any impurities; see Fig. S9 (ESI $\dagger$ ). Afterwards, high angle annular dark field (HAADF) STEM was carried out to study the sample at the atomic level; see Fig. 3.

By the combination of both STEM and an EELS analysis technique named spectrum imaging (SI), ${ }^{11}$ we can disclose the

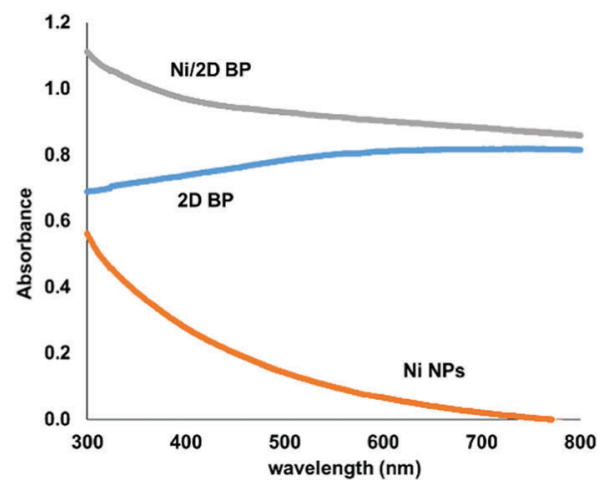

Fig. 1 UV-Vis spectra of 2D BP, Ni NPs and Ni/2D BP. 


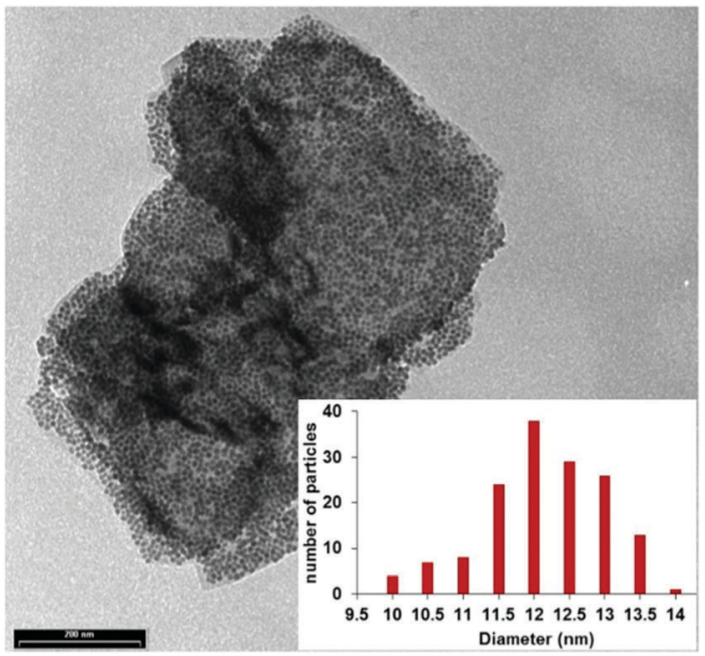

Fig. 2 TEM image of Ni NPs supported on few-layer black phosphorus, $P$ : Ni molar ratio $3: 1$. Scale bar: $200 \mathrm{~nm}$. Inset: Size distribution histogram of Ni NPs.

chemical composition of the surface of the new nanocomposite. For this purpose, we acquired the experimental $\mathrm{Ni} \mathrm{L}_{2,3}$-edge energy loss near edge structure (ELNES) for Ni NPs and NiO $\mathrm{NPs},{ }^{12}$ the latter taken as a reference sample, grey and red spectra in Fig. 4, respectively, and compared with the reference spectra from the literature, blue and purple, respectively. ${ }^{12}$ Our EELS spectra shown in Fig. 4 were extracted from the SI dataset after background subtraction and multiple scattering deconvolution, in order to avoid artifacts due to specimen thickness. Here, besides the $L_{3}$ and $L_{2}$ peaks at $855 \mathrm{eV}$ and $872 \mathrm{eV}$, respectively, a secondary peak is observed (black arrow I on grey and blue spectra). This secondary peak is present only in pure metallic $\mathrm{Ni}^{13}$ and arises from a $4 \mathrm{p}-4 \mathrm{~d}$ hybridized band ${ }^{12}$ that is absent in nickel oxide (red spectrum). These results, compared with the spectrum acquired on NiO NPs, showing a

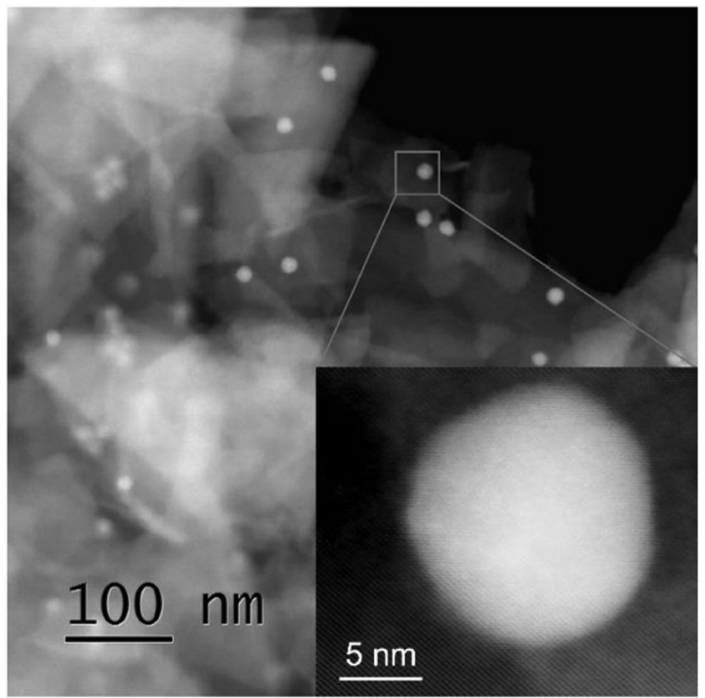

Fig. 3 HAADF-STEM of Ni/2D BP with the inset showing atomic resolution for 2D BP.

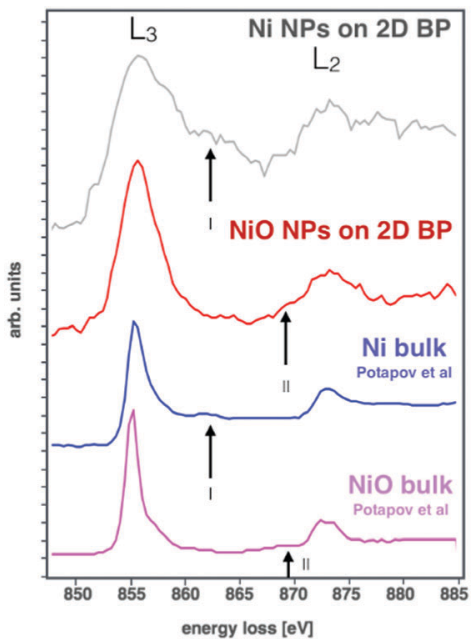

Fig. $4 \mathrm{Ni} L_{2,3}$-edge energy loss near edge structure (ELNES) for Ni/2D BP and $\mathrm{NiO} / 2 \mathrm{D} \mathrm{BP}$, and the equivalent signals from bulk structures of $\mathrm{Ni}$ and $\mathrm{NiO}{ }^{12}$

typical bump around $869 \mathrm{eV}^{12}$ (see black arrow II on red and purple spectra of Fig. 4), provide a strong confirmatory evidence that the prepared Ni NPs are not oxidized and add further evidence that the BP flakes are strictly decorated with non-oxidized metallic nickel nanoparticles. In keeping with this finding, the observed catalytic activity of the hybrid system, vide infra, confirms that no oxidation of Ni NPs has occurred.

The new nanohybrid was further characterized by Raman spectroscopy. Fig. 5 compares the Raman spectrum of a freshly prepared sample of 2D BP (blue curve) with the same batch of 2D BP after functionalization with nickel nanoparticles (red curve).

The spectrum of the nickel functionalized material reveals the three typical Raman bands of black phosphorus at 360, 435 and $460 \mathrm{~cm}^{-1}$, attributed to the $A_{g}^{1}, B_{2 g}$, and $A_{g}^{2}$ vibrational modes respectively, ${ }^{14}$ confirming the orthorhombic crystalline structure of 2D BP. Since nickel is silent in Raman, the crystalline nature of the nanoparticles was confirmed by SAED and powder XRD, see Fig. S6 and S7 (ESI†), which showed that the nanoparticles have the characteristic cubic face centred structure of crystalline nickel.

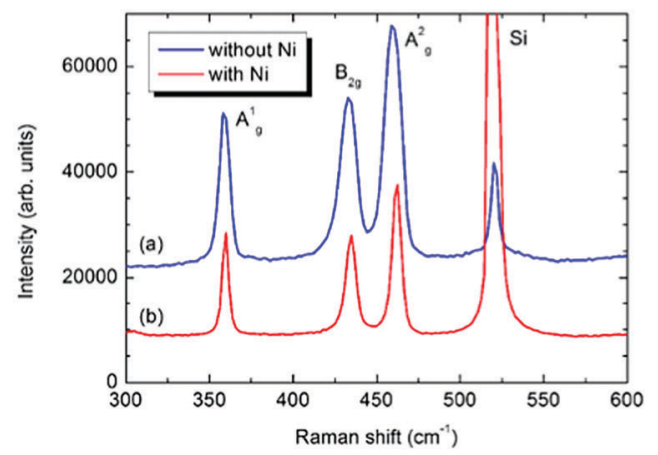

Fig. 5 Comparison of the Raman spectra between (a) pristine fewlayered BP (blue curve) and (b) Ni/2D BP (red curve). The strong peak at $520 \mathrm{~cm}^{-1}$ is due to the silicon substrate. 
a)

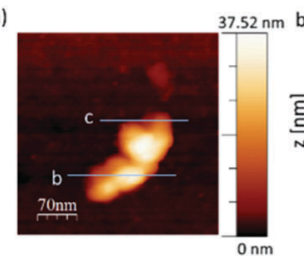

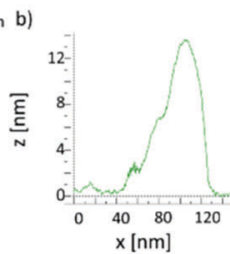

c)

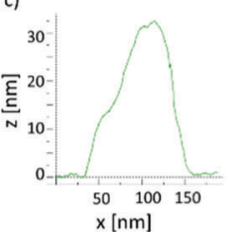

Fig. 6 (a) AFM image of a typical flake and ( $b$ and $c$ ) profiles along the two lines displayed in (a).

On the same sample AFM was measured. Looking at smaller structures, which are likely to be single flakes or few flakeaggregates, see Fig. S11 (ESI $\dagger$ ), we found their typical size to be a few hundred nanometers, and their thickness up to $50 \mathrm{~nm}$. A typical flake is shown in Fig. 6(a), and two cross-sections are shown in Fig. 6(b) and (c). Knowing that a single Ni nanoparticle has a diameter around $12 \mathrm{~nm}$, and using the same approximation as in Hersam's paper $^{8 c}$ we can roughly estimate the BP flake thickness. The object in Fig. 6(a), with a thickness of 14-32 nm, corresponds to a BP flake thickness of 2-20 nm, and having the BP layer spacing of $0.53 \mathrm{~nm}$, the number of layers ranges from 4 to 38 .

Powder X-ray diffraction of Ni/2D BP (see Fig. S10, ESI $\dagger$ ) shows the characteristic peaks of black $\mathrm{P}$ and additionally a broad peak at $2 \theta$ equal to $44.4^{\circ}$ revealing the presence of nickel NPs. The other peaks at $51.8^{\circ}$ and $76.4^{\circ}$, also characteristic of nickel, are too weak to be identified. Thus, the surface functionalization with Ni NPs did not affect the structure and the crystallinity of 2D BP.

The environmental instability of exfoliated BP has been an issue so far, since under ambient conditions few-layer BP degrades completely in less than a week, preventing its applications in several fields. ${ }^{15}$ Numerous strategies have been applied for the ambient protection of $2 \mathrm{D} \mathrm{BP}$, such as the coating with $\mathrm{Al}_{2} \mathrm{O}_{3},{ }^{16} \mathrm{MoS}_{2}{ }^{17}$ and recently with ionic liquids. ${ }^{18}$ We were interested to study the influence, if any, of the functionalization with Ni NPs. A fresh suspension of Ni/2D BP was drop-casted on a TEM grid and on a $\mathrm{SiO}_{2} / \mathrm{Si}$ support and exposed in the darkness to ambient oxygen and moisture. TEM and Raman measurements were acquired regularly over one week and in parallel, a sample of pristine 2D BP was examined as a reference. Surprisingly, while the latter was quickly degraded to molecular compounds, see Fig. S12 (ESI $\dagger$ ), the morphology of Ni/2D BP flakes was unaltered after one week as shown by TEM in Fig. 7. The Raman spectrum still clearly displayed the typical peaks of BP.

Being confident of the increased stability and aware that $2 \mathrm{D}$ BP possesses a higher surface to volume ratio than other 2D materials due to its puckered lattice configuration, ${ }^{19}$ we investigated the catalytic activity of $\mathrm{Ni} / 2 \mathrm{D} \mathrm{BP}$ in a model reaction, the semihydrogenation of phenylacetylene to styrene (Scheme 1). This is an important industrial reaction, because phenylacetylene is a poisoning impurity in styrene feedstocks that causes deactivation of the styrene polymerization catalyst.

The conversion was quantitative with high selectivity towards styrene (see Table S1, ESI $\dagger$ ), outperforming known catalytic systems based on supported nickel nanoparticles..$^{20} \mathrm{~A}$ catalytic test with bare colloidal nanoparticles was carried out, see Table S1 (ESI $\dagger$ ),
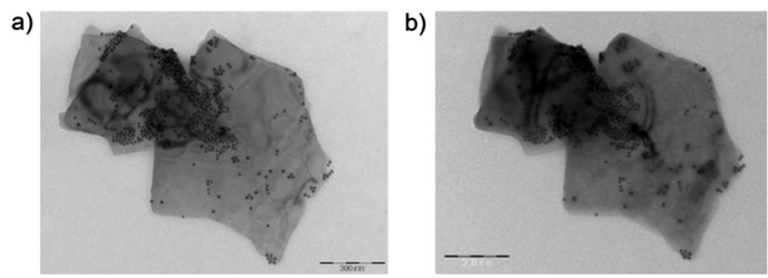

Fig. 7 Bright field TEM images of a Ni/2D BP flake observed at (a) time $=0$ and (b) after one week. Scale bar: $200 \mathrm{~nm}$.

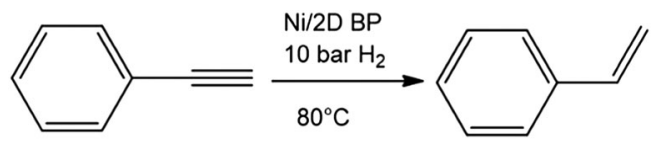

Scheme 1 Semihydrogenation of phenylacetylene to styrene.

and interestingly there was a drop from $92.8 \%$ to $78.6 \%$ in selectivity to styrene versus the test carried out with Ni/2D BP. Pristine few-layered BP was also tested and, as expected, did not show any catalytic activity.

The catalyst Ni/2D BP could be easily separated by centrifugation and its reusability in the same process was tested. The conversion was quantitative up to the fourth run, and the selectivity remains unaltered, as shown in Fig. 8. TEM inspection of Ni/2D BP recovered after the catalytic tests confirmed the presence of Ni NPs on the surface of the nanosheets, with an averaged dimension of $11.5 \pm$ $0.9 \mathrm{~nm}$, and as before starting the catalysis, the BP flakes looked unchanged as well, see Fig. S13 (ESI $\dagger$ ). In comparison, unsupported Ni NPs lose their activity just after the first cycle. ${ }^{21}$ These results indicate a strong metal-support interaction that gives high stability to nickel nanoparticles thus preventing their aggregation and leaching during the consecutive catalytic runs.

Leaching tests were carried out to verify the presence in solution of both nickel and molecular compounds containing phosphorus, which might be possibly formed from the degradation of $2 \mathrm{D}$ BP during the process. Analysis by inductively coupled plasma mass spectrometry (ICP-MS) of the reaction mixture after catalyst separation showed that both $\mathrm{Ni}$ and $\mathrm{P}$ were absent at the ppm level, meaning Ni NPs are strongly immobilized on the surface of 2D BP which consequently acts as a suitable support and does not degrade to phosphorus oxyacids under catalytic conditions. Therefore, the

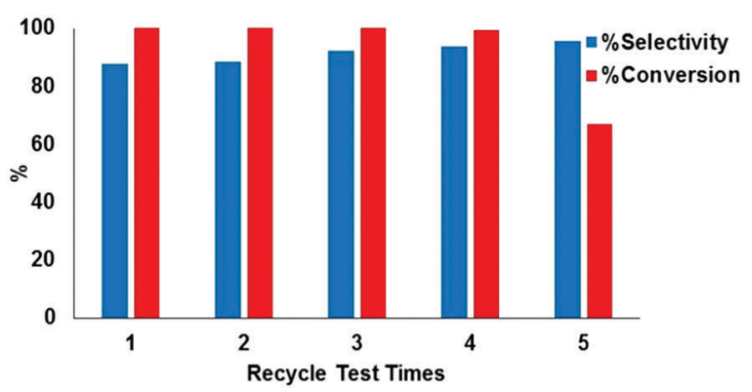

Fig. 8 Recycling tests. Reaction conditions: 10 bar $\mathrm{H}_{2}, 80^{\circ} \mathrm{C}, 2$ hours, $\mathrm{Ni}$ $\mathrm{NPS}=1.2 \mathrm{~mol} \%$, phenylacetylene $=0.3 \mathrm{mmol}, 1.41 \mathrm{mg}$ catalyst (15.2 wt\% $\mathrm{Ni}$. Both conversion and selectivity were evaluated using GC and GC-MS. 
observed decrease of the catalytic efficiency in the fifth run (67\%) might be reasonably ascribed to nickel oxidation during work-up.

In summary, a new surface functionalization of exfoliated BP has been carried out with Ni NPs. Morphological analysis of the nanohybrid by AFM and electron microscopy was carried out and reveals that the nanoparticles are homogeneously dispersed on the flakes' surface. Raman and XRD confirmed the nature and crystallinity of the material. A detailed study of the environmental stability of the new 2D material was carried out by means of TEM and Raman and resulted in an enhanced stability under ambient conditions towards the degradation to P-oxides and acids. Taking advantage of these properties, $\mathrm{Ni} / 2 \mathrm{D}$ $\mathrm{BP}$ was tested as a reusable catalyst in the semihydrogenation of phenylacetylene, where it worked successfully exhibiting good conversion and selectivity to styrene in successive cycles, which is remarkable since known catalysts based on Ni NPs are less selective. Finally, we anticipate that the decoration of the surface of BP flakes with NPs may induce peculiar electronic and adsorption properties in comparison to pristine $\mathrm{BP}$, which could be exploited for the fabrication of new electronic devices as, for instance, next-generation gas sensors.

This work was financially supported by EC through the project PHOSFUN "Phosphorene functionalization: a new platform for advanced multifunctional materials" (ERC ADVANCED GRANT No. 670173 to M. P.). F. T. thanks CNR-NANO for funding the SEED project SURPHOS. S. H. acknowledges support from Scuola Normale Superiore, project SNS16_B_HEUN - 004155. Thanks are expressed also to Italian Ministry of Education and Research (MIUR) under the project Beyond-Nano (PON a3_00363).

\section{Conflicts of interest}

There are no conflicts to declare.

\section{Notes and references}

1 (a) E. S. Reich, Nature, 2014, 506, 19; (b) H. O. H. Churchill and P. Jarillo-Herrero, Nat. Nanotechnol., 2014, 9, 330.

2 (a) L. Kou, C. Chen and S. C. Smith, J. Phys. Lett., 2015, 6, 2794; (b) S. Balendhran, S. Walia, H. Nili, S. Sriram and M. Bhaskaran, Small, 2015, 11, 640.

3 (a) K. Li, Y. J. Yu, G. J. Ye, Q. Q. Ge, X. D. Ou, H. Wu, D. L. Feng, X. H. Chen and Y. B. Zhang, Nat. Nanotechnol., 2014, 9, 372; (b) H. Liu, A. T. Neal, Z. Zhu, Z. Luo, X. Xu, D. Tomanek and P. D. Ye, ACS Nano, 2014, 8, 4033.

4 (a) J. Kang, J. D. Wood, S. A. Wells, J.-H. Lee, X. Liu, K.-S. Chen and M. C. Hersam, ACS Nano, 2015, 9, 3596; (b) P. Yasaei, B. Kumar, T. Foroozan, C. Wang, M. Asadi, D. Tuschel, J. E. Indacochea, R. F. Klie and A. Salehi-Khojin, Adv. Mater., 2015, 27, 1887; (c) A. H. Woomer, T. W. Farnsworth, J. Hu, R. A. Wells, C. L. Donley and S. C. Warren, ACS Nano, 2015, 9, 8869; (d) H. Wang, X. Yang, W. Shao, S. Chen, J. Xie, X. Zhang, J. Wang and Y. Xie, J. Am. Chem. Soc., 2015, 137, 11376; (e) Z. Guo, H. Zhang, S. Lu, Z. Wang, S. Tang, J. Shao, Z. Sun, H. Xie, H. Wang, X.-F. Yu and P. K. Chu, Adv. Funct. Mater., 2015, 25, 6996; ( $f$ ) Z. Sun, H. Xie, S. Tang, X.-F. Yu, Z. Guo, J. Shao, H. Zhang, H. Huang, H. Wang and P. K. Chu, Angew. Chem., Int. Ed., 2015, 54, 11526; (g) E. A. Lewis, J. R. Brent, B. Derby, S. J. Haigh and D. J. Lewis, Chem. Commun., 2017, 53, 1445 and references therein.

5 M. Serrano-Ruiz, M. Caporali, A. Ienco, V. Piazza, S. Heun and M. Peruzzini, Adv. Mater. Interfaces, 2016, 3, 1500441.

6 L. Li, F. Yang, G. J. Ye, Z. Zhang, Z. Zhu, W. Lou, X. Zhou, L. Li, K. Watanabe, T. Taniguchi, K. Chang, Y. Wang, X. H. Chen and Y. Zhang, Nat. Nanotechnol., 2016, 11, 593.

7 (a) S. Das, W. Zhang, M. Demarteau, A. Hoffmann, M. Dubey and A. Roelofs, Nano Lett., 2014, 14, 5733; (b) V. Tran, R. Soklaski, Y. Liang and L. Yang, Phys. Rev. B: Condens. Matter Mater. Phys., 2014, 89, 235319; (c) A. Castellanos-Gomez, J. Phys. Chem. Lett., 2015, 6, 4280.

8 (a) H. U. Lee, S. C. Lee, J. Won, B.-C. Son, S. Choi, Y. Kim, S. Y. Park, H.-S. Kim, Y.-C. Lee and J. Lee, Sci. Rep., 2015, 5, 8691; (b) Y. Zhao, H. Huang, Q. Xiao, Y. Hu, Z. Guo, H. Xie, J. Shao, Z. Sun, W. Han, X. F. Yu, P. Li and P. K. Chu, Angew. Chem., Int. Ed., 2016, 55, 5003; (c) C. R. Ryder, J. D. Wood, S. A. Wells, Y. Yang, D. Jariwala, T. J. Marks, G. C. Schatz and M. C. Hersam, Nat. Chem., 2016, 8, 597; (d) G. Abellan, V. Lloret, U. Mundloch, M. Marcia, C. Neiss, A. Görling, M. Varela, F. Hauke and A. Hirsch, Angew. Chem., Int. Ed., 2016, 55, 14557.

9 (a) V. V. Kulish, O. I. Malyi, C. Persson and P. Wu, Phys. Chem. Chem. Phys., 2015, 17, 992; (b) T. Hu and J. Hong, J. Phys. Chem. C, 2015, 119, 8199; (c) Y. Jing, X. Zhang and Z. Zhou, Wiley Interdiscip. Rev.: Comput. Mol. Sci., 2016, 6, 5; (d) P. Rastogi, S. Kumar, S. Bhowmick, A. Agarwal and Y. S. Chauhan, 2015, arXiv:1503.04296v2 [condmat.mes-hall]; (e) L. Seixas, A. Carvalho and A. H. Castro-Neto, Phys. Rev. B: Condens. Matter Mater. Phys., 2015, 91, 155138; $(f)$ X. Sui, C. Si, B. Shao, X. Zou, J. Wu, B. L. Gu and W. Duan, J. Phys. Chem. C, 2015, 119, 10059; $(g)$ H. Wang, S. Zhu, F. Fan, Z. Li and H. Wu, J. Magn. Magn. Mater., 2016, 401, 706.

10 S. Carenco, C. Boissière, L. Nicole, C. Sanchez, P. Le Floch and N. Mézailles, Chem. Mater., 2010, 22, 1340.

11 G. Nicotra, Q. M. Ramasse, I. Deretzis, A. La Magna, C. Spinella and F. Giannazzo, ACS Nano, 2013, 7, 3045.

12 Nickel(0) nanoparticles, obtained as described in ESI, $\uparrow$ were exposed for one week to air at ambient conditions to allow the complete oxidation. The resulting NiO NPs were immobilized on 2D BP following the same procedure used for the preparation of Ni/2D BP.

13 (a) P. L. Potapov, S. E. Kulkova, D. Schryvers and J. Verbeeck, Phys. Rev. B: Condens. Matter Mater. Phys., 2001, 64, 184110; (b) R. D. Leapman, L. A. Grunes and P. L. Fejes, Phys. Rev. B: Condens. Matter Mater. Phys., 1982, 26, 614.

14 (a) S. Liu, N. Huo, S. Gan, Y. Li, Z. Wei, B. Huang, J. Liu, J. Li and H. Chen, J. Mater. Chem. C, 2015, 3, 10974; (b) J. Lin, L. Liang, X. Ling, S. Zhang, N. Mao, N. Zhang, B. G. Sumpter, V. Meunier, L. Tong and J. Zhang, J. Am. Chem. Soc., 2015, 137, 15511; (c) Y. Feng, J. Zhou, Y. Du, F. Miao, C. G. Duan, B. Wang and X. Wan, J. Phys.: Condens. Matter, 2015, 27, 185302.

15 J. O. Island, G. A. Steele, H. S. van der Zant and A. CastellanosGomez, 2D Mater., 2015, 2, 011002.

16 J. D. Wood, S. A. Wells, D. Jariwala, K.-S. Chen, E. Cho, V. K. Sangwan, X. Liu, L. J. Lauhon, T. J. Marks and M. C. Hersam, Nano Lett., 2014, 14, 6964.

17 Y. Son, D. Kozawa, A. Tianxiang Liu, V. B. Koman, Q. H. Wang and M. S. Strano, 2D Mater., 2017, 4, 025091.

18 S. Waalia and V. Bansal, et al., Adv. Mater., 2017, 1700152.

19 S.-Y. Cho, Y. Lee, H. J. Koh, H. Jung, J.-S. Kim, H.-W. Yoo, J. Kim and H.-T. Jung, Adv. Mater., 2016, 28, 7020.

20 (a) Y. Chen, C. Li, J. Zhou, S. Zhang, D. Rao, S. He, M. Wei, D. G. Evans and X. Duan, ACS Catal., 2015, 5, 5756; (b) C. Li, Y. Chen, J. Zhou, S. Zhang, S. He, M. Wei, D. G. Evans and X. Duan, ChemCatChem, 2014, 6, 824; $(c)$ A. V. Erokhin, E. S. Lokteva, A. Ye. Yermakov, D. W. Boukhvalov, K. I. Maslakov, E. V. Golubina and M. A. Uimin, Carbon, 2014, 74, 291; (d) E. V. Golubina, E. S. Lokteva, A. V. Erokhin, A. A. Velighzanin, Y. V. Zubavichus, V. A. Likholobov and V. V. Lunin, J. Catal., 2016, 344, 90.

21 J. Park, E. Kang, S. U. Son, H. M. Park, M. K. Lee, J. Kim, K. W. Kim, H.-J. Noh, J.-H. Park, C. J. Bae, J.-G. Park and T. Hyeon, Adv. Mater., $2005,17,429$. 